hep-th/0402185

DAMTP-2004-9

DESY-04-024

\title{
On the plane-wave cubic vertex
}

\author{
James Lucietti ${ }^{b}$, Sakura Schäfer-Nameki ${ }^{\sharp}$ and Aninda Sinha ${ }^{b}$ \\ ${ }^{\mathrm{D}}$ DAMTP, University of Cambridge \\ Wilberforce Road, Cambridge CB3 OWA, U.K. \\ \#II. Institut für Theoretische Physik, University of Hamburg \\ Luruper Chaussee 149, 22761 Hamburg, Germany
}

\begin{abstract}
The exact bosonic Neumann matrices of the cubic vertex in plane-wave light-cone string field theory are derived using the contour integration techniques developed in our earlier paper. This simplifies the original derivation of the vertex. In particular, the Neumann matrices are written in terms of $\mu$-deformed Gamma-functions, thus casting them into a form that elegantly generalizes the well-known flat-space solution. The asymptotics of the $\mu$-deformed Gamma-functions allow one to determine the large- $\mu$ behaviour of the Neumann matrices including exponential corrections. We provide an explicit expression for the first exponential correction and make a conjecture for the subsequent exponential correction terms.
\end{abstract}

$2 / 2004$

Email: J.Lucietti, S.Schafer-Nameki, A.Sinha@damtp.cam.ac.uk 


\section{Introduction}

The BMN-correspondence [1] between the plane-wave limit of IIB string theory on $A d S_{5} \times S^{5}[2,3]$ and a certain sector of the $d=4, \mathcal{N}=4$ SYM theory has proven to be a powerful means in paving the way towards an improved understanding of the AdS/CFT correspondence.

Despite the impressive advancements in the analysis of both sides of this correspondence, some key questions still remain to be answered. One central point that has so far not been addressed to full satisfaction is the study of interactions and their dual interpretation. For instance, given the exact cubic vertex, the string scattering amplitudes can in principle be calculated for all values of $\mu$. For large $\mu$ this would allow comparison with perturbative gauge theory calculations and moreover the knowledge of finite $\mu$ corrections could provide very interesting predictions for finite $\lambda^{\prime}$ corrections in the gauge theory.

Some important results in this direction have already been obtained. On the string theory side, progress has been made towards understanding the interactions in the framework of light-cone string field theory. This was first developed for flat space in [4,5,6] and then generalized to the plane-wave in $[7,8,9,10,11,12,13,14]$. For comparisons to the gauge theory side see e.g. [15, 16, 17, 1]. However, quantities that have been computed for all $\mu$ are still rather rare, and we believe comprise only the results of [1] for the cubic closed interaction vertex as well as [14] for the open-closed vertex. Furthermore, in order to perform computations of scattering amplitudes based on these results, it would be advantageous if the interactions are expressed in a concise form reminiscent of the flat-space results.

The main motivation for the present paper is to re-address the analysis of the planewave cubic vertex. The derivation that we shall provide for the vertex relies on the contour method for summing certain infinite series, which was developed in [14]. One of the main merits of this approach is that it allows a derivation very close to the one known for the flat space vertex in [5]. The solution to the cubic vertex equation, as in [14], will be written in terms of $\mu$-deformed Gamma-functions. This gives an elegant generalization of the flat-space solutions of [5].

There is a slight discrepancy between the explicit expression of the Neumann matrices in [11] and ours. It appears that the only problem in [11] is the final expression for the Neumann vectors (i.e., their equation (52) 2). We derive the large $\mu$ asymptotics directly

1 For a more complete list of references see $18,19,20,21,22$.

2 In fact it is easy to see that there is some error in (52) of [1]; simply note that $\phi_{m 3}$ in the equation in question is divergent. 
from the exact expression for the Neumann vectors, which agree with those in [11]. Despite the discrepancy in the exact expression, this is not surprising since the asymptotics in [11] were not developed from their exact expression for the vertex. We will elaborate on this in section 3.3. We also extend the large- $\mu$ asymptotics by explicitly computing the first exponential corrections and provide a conjecture for the subsequent exponential correction terms.

The plan of this paper is as follows. In section 2 we present the derivation of the cubic vertex in flat space using the contour method for summing series of [14]. In section 3 we generalize this to the cubic vertex in the plane-wave. In section 4 we use the asymptotics of the $\mu$-deformed Gamma-functions to derive the large- $\mu$ expansions of the Neumann matrices. We also comment upon the exponential corrections appearing in the large- $\mu$ asymptotic expansions and explicitly give the first term, as well as provide a conjecture for the subsequent terms. We conclude in section 5. There are four appendices, in which various properties and asymptotics of the $\mu$-deformed Gamma-functions are derived.

\section{The cubic vertex in flat-space}

In this section, as a warm-up, we will use the contour method of [14] in order to derive the well-known flat-space Neumann matrices $\bar{N}_{m n}^{r s}$ for the cubic string vertex, which originally were determined in [5]. We shall focus on the bosonic part of the vertex, for which the standard ansatz is

$$
|V\rangle=\mathcal{N} \exp \left(\frac{1}{2} \sum_{r, s=1}^{3} \sum_{m, n=1}^{\infty} a_{-m}^{(r)} \bar{N}_{m n}^{r s} a_{-n}^{(s)}+\sum_{r=1}^{3} \sum_{m=1}^{\infty} a_{-m}^{(r)} \bar{N}_{m}^{r} \mathbf{P}+K \mathbf{P}^{2}\right)|0\rangle,
$$

where $a_{-m}^{(r)}$ are the normalized oscillators of the $r$-th string and $\mathbf{P}^{i}=2 p_{1}^{+} p_{2}^{i}-2 p_{2}^{+} p_{1}^{i}$. Geometrical continuity conditions and momentum conservation then imply constraints upon the Neumann matrices. It was shown that the Neumann matrices satisfy

$$
\bar{N}_{m n}^{r s}=-\frac{m n \alpha_{1} \alpha_{2} \alpha_{3}}{n \alpha_{r}+m \alpha_{s}} \bar{N}_{m}^{r} \bar{N}_{n}^{s}
$$

where

$$
\bar{N}_{m}^{r}=-\left(\left(A^{(r)}\right)^{t} \Gamma^{-1} B\right)_{m}, \quad K=-\frac{1}{4} B \Gamma^{-1} B,
$$

and $\alpha_{i}=2 p^{(i)+}$ are the momenta of the various strings, which are chosen such that $\sum_{i=1}^{3} \alpha_{i}=0$, and $\tau_{0}=\sum_{r=1}^{3} \alpha_{r} \log \left|\alpha_{r}\right|$. Without loss of generality we assume $\alpha_{1}, \alpha_{2}>0$ 
and $\alpha_{3}<0$. The matrices $A$ and $B$ are defined in appendix $\mathrm{A}$ and correspond to various Fourier modes. Further

$$
\Gamma=\sum_{r=1}^{3} A^{(r)}\left(A^{(r)}\right)^{t} .
$$

To solve for the Neumann matrices, it is clear that it is sufficient to determine $\Gamma^{-1} B$. This is most conveniently done by solving the two coupled series

$$
\begin{aligned}
\sum_{n=1}^{\infty} \sqrt{n} \bar{f}_{n}^{(3)} A_{n m}^{(r)} & =\frac{\alpha_{3}}{\alpha_{r}} \sqrt{m} \bar{f}_{m}^{(r)} \\
\sum_{r=1}^{3} \sum_{n=1}^{\infty} \frac{\sqrt{n}}{\alpha_{r}} A_{m n}^{(r)} \bar{f}_{n}^{(r)} & =-B_{m},
\end{aligned}
$$

for $\bar{f}_{n}^{(r)}$. These are related to the Neumann vectors by

$$
\bar{f}_{m}^{(r)}=\frac{\alpha_{r}}{\sqrt{m}} \bar{N}_{m}^{r}
$$

An additional constraint is

$$
\sum_{n=1}^{\infty} \sqrt{n} \bar{f}_{n}^{(3)} B_{n}=-2 \frac{\tau_{0}}{\alpha_{1} \alpha_{2}},
$$

which comes from the knowledge of the explicit form of $K$ in flat-space 3 . The equations (2.5) are equivalent to

$$
\begin{gathered}
\sum_{m=1}^{\infty} \frac{(-1)^{m}}{m} \sin (m \pi \beta) \bar{f}_{m}^{(3)}=\pi \frac{\tau_{0}}{\alpha_{3}} \\
\sum_{m=1}^{\infty}(-1)^{m+n} m \frac{\sin (m \pi \beta)}{n^{2}-m^{2} \beta^{2}} \bar{f}_{m}^{(3)}=-\frac{\pi}{2 \beta^{2}} \bar{f}_{n}^{(1)} \\
\sum_{m=1}^{\infty}(-1)^{m} m \frac{\sin (m \pi \beta)}{n^{2}-m^{2}(1+\beta)^{2}} \bar{f}_{m}^{(3)}=\frac{1}{(\beta+1)^{2}} \frac{\pi}{2} \bar{f}_{n}^{(2)} \\
\frac{\beta}{\alpha_{1}} \sum_{n=1}^{\infty} n(-1)^{n} \frac{\bar{f}_{n}^{(1)}}{n^{2}-m^{2} \beta^{2}}+\frac{1+\beta}{\alpha_{2}} \sum_{n=1}^{\infty} n \frac{\bar{f}_{n}^{(2)}}{n^{2}-m^{2}(1+\beta)^{2}}(-1)^{m} \frac{1}{\sin (m \pi \beta)} \bar{f}_{m}^{(3)}-\frac{\alpha_{3}}{\alpha_{1} \alpha_{2}} \frac{1}{m^{2}} .
\end{gathered}
$$

3 Note, that the RHS of this equation equals $K$, which in flat-space can be determined using conformal invariance, whereby one maps the known Neumann functions of the complex plane to the light-cone string diagram, $c f$. [23]. Due to the absence of explicit conformal invariance in the light-cone gauge, this is not possible anymore in the plane-wave, so that the condition (2.7) cannot be used to derive properties of $f^{(3)}$. This will be discussed in the next section. 
Here, we defined $\beta=\alpha_{1} / \alpha_{3}<0$.

We shall now apply the contour method in order to derive the solutions to these equations. Schematically, the contour method provides one with solutions $f(n)$ to certain coupled series $\sum_{n} f(n)=F$ for given $F$ (note these can be interpreted as infinite dimensional matrix equations). The main idea is to map the sum to a contour integral in the plane so that by Cauchy's theorem

$$
\sum_{n=1}^{\infty} f(n)+\sum_{k} \operatorname{Res}_{z=z_{k}} \pi \cot (\pi z) f(z)=\lim _{R \rightarrow \infty} \oint_{C_{R}} \frac{d z}{2 \pi i} \pi \cot (\pi z) f(z),
$$

where $C_{R}$ is the contour given by a circle of radius $R$ centred on the origin, not intersecting any poles of the integrand (so in particular $R \neq 1,2,3 \ldots$ ). If the RHS of (2.12) vanishes, one can compare the sum over residues with $F$, which allows one to infer the poles and zeroes of $f(z)$ (note we will assert that $f(z)=0$ for $z=0,-1,-2 \ldots$ ). For more details and examples on the contour method we refer the reader to [14].

Applied to the present context, i.e., in order to find the solutions for $\bar{f}_{n}^{(r)}$, we map the sum to a contour integral in the complex plane, i.e., consider in view of (2.8)

$$
\begin{aligned}
\oint \frac{d m}{2 \pi i} \pi \cot (\pi m) & \frac{1}{\cos (\pi m)} \frac{\sin (m \pi \beta)}{m} \bar{f}^{(3)}(m) \\
& =-\oint \frac{d m}{2 \pi i} \Gamma(m+1) \Gamma(-m) \frac{\sin (m \pi \beta)}{m} \bar{f}^{(3)}(m)
\end{aligned}
$$

where we have rewritten $(-1)^{m}=1 / \cos (\pi m)$. Assume that there are no relative cancellations of residues, and that the contribution to the sum comes from a single residue. The sum (2.8) arises from the poles at $m \in \mathbb{N}$. In order to cancel the poles of $\Gamma(m+1)$ for $m \in \mathbb{Z}_{-}, \bar{f}^{(3)}(m)$ needs to have a factor $1 / \Gamma(m+1)$. Further, it can have poles at $m \beta \in \mathbb{Z}_{-}$ or $m \beta \in \mathbb{Z}_{+}$, i.e., $\bar{f}_{m}^{(3)} \propto \Gamma( \pm \beta m)$. In fact, it has to have such a factor, as otherwise, evaluating the contour integral for (2.9) would imply that $\bar{f}_{n}^{(1)}$ vanishes, which is unphysical. Further, since $\beta<0$ the only consistent choice is that the poles are at $m \beta \in \mathbb{Z}_{+}$, as otherwise $\bar{f}_{m}^{(3)}$ would be singular for the particular value $\beta=-1$. In summary we deduced that

$$
\bar{f}^{(3)}(m)=\hat{f}^{(3)}(m) \frac{\Gamma(-m \beta)}{\Gamma(m+1)},
$$

with $\hat{f}^{(3)}(m)$ having no poles at $m \in \mathbb{Z}$ or $m \beta \in \mathbb{Z}$.

In evaluating the integral for (2.10)

$$
\oint \frac{d m}{2 \pi i} m \frac{\Gamma(-m)}{\Gamma(1+m \beta)} \frac{\pi}{n^{2}-m^{2}(1+\beta)^{2}} \hat{f}_{m}^{(3)},
$$


one obtains poles at $m= \pm n /(1+\beta)$. Assuming that the contributions to the sums come only from one term, implies that $\hat{f}_{m}^{(3)}$ has to have zeroes at all values $m$, such that $\eta m(1+\beta) \in \mathbb{Z}_{+}$for one of the signs $\eta= \pm 1$. Thus, we may make the further ansatz

$$
\hat{f}_{m}^{(3)}=\frac{1}{\Gamma(-\eta m(1+\beta)+1)} \check{f}_{m}^{(3)}
$$

For the choice $\eta=1$ 国, the residue of (2.15) at $m=-n /(1+\beta)$ implies

$$
\bar{f}_{n}^{(2)}=\frac{\Gamma\left(\frac{n}{1+\beta}\right)}{\Gamma\left(1-n\left(\frac{\beta}{1+\beta}\right)\right) \Gamma(n+1)} \check{f}_{-\frac{n}{1+\beta}}^{(3)}=\frac{\Gamma\left(-n \frac{\alpha_{3}}{\alpha_{2}}\right)}{\Gamma\left(-n\left(\frac{\alpha_{3}}{\alpha_{2}}+1\right)+1\right) \Gamma(n+1)} \check{f}_{n \frac{\alpha_{3}}{\alpha_{2}}}^{(3)} .
$$

The contour argument applies only if the integrand suitably falls off at infinity (cf. [14]). Invoking Stirling's formula, the $m$-dependent part of the integrand in (2.15) has an asymptotic behaviour given by

$$
\begin{aligned}
m^{-5 / 2} \beta^{m \beta}(1+\beta)^{m(1+\beta)} \check{f}^{(3)}(m) & =m^{-5 / 2} \alpha_{1}^{-m \frac{\alpha_{1}}{\alpha_{3}}} \alpha_{2}^{-m \frac{\alpha_{2}}{\alpha_{3}}}\left(-\alpha_{3}\right)^{-m} \check{f}^{(3)}(m) \\
& =m^{-5 / 2} e^{-m \tau_{0} / \alpha_{3}} \check{f}^{(3)}(m) .
\end{aligned}
$$

Thus, $\check{f}^{(3)}$ must contain a factor $e^{m \tau_{0} / \alpha_{3}}$, but could in principle also be proportional to $\sum_{k} a_{k} m^{k}$ for some suitable powers $k$, which respect the required asymptotics. The same asymptotic behaviour is obtained for the integrands for (2.8) and (2.10). We shall fully determine $\check{f}$ below.

With the new ansatz (2.16), the residue for the sum in (2.8) becomes

$$
\sum_{m=1}^{\infty} \frac{(-1)^{m}}{m} \sin (m \pi \beta) \bar{f}_{m}^{(3)}=\left.\pi \frac{d \check{f}^{(3)}(m)}{d m}\right|_{m=0}
$$

so that

$$
\left.\frac{d \check{f}^{(3)}(m)}{d m}\right|_{m=0}=\frac{\tau_{0}}{\alpha_{3}} .
$$

Now consider the integral for equation (2.9),

$$
(-1)^{n} \oint \frac{d m}{2 \pi i} \frac{\pi \cot (\pi m)}{\cos (\pi m)} m \frac{\sin (m \pi \beta)}{n^{2}-m^{2} \beta^{2}} \frac{\Gamma(-m \beta)}{\Gamma(m+1)} \hat{f}^{(3)}(m)
$$

4 With the choice $\eta=-1$, one would encounter a pole at some positive real integer for some choice of $\beta$. The non-vanishing contributions from this pole would lead to inconsistencies. 
The only pole that has a non-trivial residue is located at $m=n / \beta$, which results in

$$
(-1)^{n+1} \frac{\pi}{2 \beta^{2}} \frac{\Gamma\left(-\frac{n}{\beta}\right)}{\Gamma(n+1)} \hat{f}_{\frac{n}{\beta}}^{(3)}=\frac{\pi}{2 \beta^{2}} \bar{f}_{n}^{(1)} .
$$

With (2.16), the function $\bar{f}^{(1)}$ is now determined as

$$
\bar{f}_{n}^{(1)}=(-1)^{n+1} \frac{\Gamma\left(-\frac{n}{\beta}\right)}{\Gamma\left(-\frac{n}{\beta}-n+1\right) \Gamma(n+1)} \check{f}_{\frac{n}{\beta}}^{(3)}=\frac{\Gamma\left(-\frac{\alpha_{2}}{\alpha_{1}} n\right)}{\Gamma\left(-n\left(\frac{\alpha_{2}}{\alpha_{1}}+1\right)+1\right) \Gamma(n+1)} \check{f}_{n \frac{\alpha_{3}}{\alpha_{1}}}^{(3)},
$$

where the reflection identity has been applied. In order to further constrain the function $\check{f}$ we need to discuss the last equation (2.11). Consider the term that gives rise to the first term in (2.11), involving $\bar{f}_{n}^{(1)}$

$$
-\oint \frac{d n}{2 \pi i} n \frac{\check{f}(3)\left(\frac{n}{\beta}\right) \Gamma(-n)}{n^{2}-m^{2} \beta^{2}} \frac{\Gamma\left(n+\frac{n}{\beta}\right)}{\Gamma\left(1+\frac{n}{\beta}\right)} .
$$

The poles are determined as in appendix $\mathrm{E}$ of [5]. At $n=0$ the pole is

$$
\operatorname{Res}_{n=0}=-\frac{\check{f}(3)(0)}{\beta(1+\beta) m^{2}} .
$$

At $n=m \beta$ the residue is

$$
\operatorname{Res}_{n=m \beta}=-\check{f}^{(3)}(m) \frac{\Gamma(-\beta m) \Gamma((1+\beta) m)}{2 \Gamma(1+m)},
$$

and for $n \in \beta /(1+\beta) \mathbb{Z}_{-}$the residues give rise to the sum

$$
-\sum_{k=1}^{\infty} \check{f}^{(3)}\left(-\frac{k}{1+\beta}\right) \frac{1}{k^{2}-m^{2}(\beta+1)^{2}} \frac{\Gamma\left(\frac{k}{\beta+1}\right)}{\Gamma(k) \Gamma\left(1-\frac{k \beta}{1+\beta}\right)} .
$$

Comparison with (2.11) yields the following additional condition on $\check{f}^{(3)}$

$$
\frac{\check{f}^{(3)}(0)}{\alpha_{1}(1+\beta) m^{2}}=-\frac{\alpha_{3}}{\alpha_{1} \alpha_{2} m^{2}},
$$

so that

$$
\check{f}^{(3)}(0)=1 .
$$

In order to fully determine $\check{f}$, recall that from the asymptotical behaviour in (2.18) we deduced that $\check{f}^{(3)}(m)=\sum_{k} a_{k} m^{k} e^{m \tau_{0} / \alpha_{3}}$. Now, (2.29) and (2.20) imply that $a_{0}=1$ and 
$a_{1}=0$. Since any higher power of $k$ would alter the asymptotics such that the contour method would not be applicable anymore, we conclude that

$$
\check{f}^{(3)}(m)=e^{m \tau_{0} / \alpha_{3}} .
$$

This is in agreement with [5]. In summary, the solutions to the equations (2.8)-(2.11) take the general form

$$
\bar{f}_{m}^{(r)}=\check{f}_{m}^{(r)} \frac{\Gamma\left(-m \frac{\alpha_{r+1}}{\alpha_{r}}\right)}{\Gamma(m+1) \Gamma\left(-m\left(\frac{\alpha_{r+1}}{\alpha_{r}}+1\right)+1\right)},
$$

where we set

$$
\check{f}_{m}^{(r)}=\check{f}_{m \frac{\alpha_{3}}{\alpha_{r}}}^{(3)}=e^{m \tau_{0} / \alpha_{r}} .
$$

In particular, (2.8)-(2.11) imply that the Neumann vectors are given by

$$
-\frac{1}{\alpha_{r}} \sqrt{m} \bar{f}_{m}^{(r)}=\left(\left(A^{(r)}\right)^{t} \Gamma^{-1} B\right)_{m}=-\bar{N}_{m}^{r}
$$

which completes the contour method derivation for the flat-space cubic vertex.

\section{The cubic vertex for the plane-wave}

Having illustrated the contour method, we are now ready to apply it to derive the cubic vertex for the plane-wave string theory. Again, we are interested in the bosonic Neumann coefficients. As in the case of the open-closed vertex derived in [14, the cubic vertex will turn out to be most concisely expressed in terms of $\mu$-deformed Gamma-functions. In particular, the following functions will be useful

$$
\begin{aligned}
\Gamma_{\mu}^{(r)}(z) & =e^{-\gamma \alpha_{r} \omega_{z}} \frac{1}{\alpha_{r} z} \prod_{n=1}^{\infty}\left(\frac{n}{\omega_{r n}+\alpha_{r} \omega_{z}} e^{\frac{\alpha_{r} \omega_{z}}{n}}\right) \\
& =\Gamma_{2 \mu \alpha_{r}}^{I}\left(\alpha_{r} z\right),
\end{aligned}
$$

where $\omega_{r n}=\sqrt{n^{2}+\alpha_{r}^{2} \mu^{2}}$ and $\Gamma_{\mu}^{I}(z)$ is the $\mu$-deformed Gamma-function defined in [14] (except $n$ as opposed to $\omega_{n}$ appears in the denominator of the infinite product). We shall define the Gamma-function without a superscript

$$
\Gamma_{\mu}(z)=\Gamma_{\mu}^{(r)}(z), \quad \text { for } \alpha_{r}=1 .
$$

A key property of these functions is that they satisfy a generalization of the reflection identity of the Gamma-function

$$
\Gamma_{\mu}^{(r)}(z) \Gamma_{\mu}^{(r)}(-z)=-\frac{\pi}{\alpha_{r} z \sin \left(\pi \alpha_{r} z\right)} .
$$

Various properties of these functions, such as asymptotics in $z$ and in $\mu$ are discussed in the appendices and in [14]. 


\subsection{Vertex equations}

The ansatz for the bosonic part of the plane-wave cubic vertex is as in (2.1). The conditions on the Neumann matrices in the plane-wave case have been derived in [7], which again reduce to the problem of finding $f_{m}^{(3)}$ (denoted by $Y_{m}$ in [11]) such that

$$
\sum_{n=1}^{\infty}\left(\Gamma_{+}\right)_{m n} f_{n}^{(3)}=\sum_{r=1}^{3} \sum_{n=1}^{\infty}\left(A^{(r)} U^{(r)}\left(A^{(r)}\right)^{t}\right)_{m n} f_{n}^{(3)}=B_{m}
$$

where

$$
\left(U^{(r)}\right)_{m n}=\delta_{m n} \frac{\left(\omega_{r m}-\alpha_{r} \mu\right)}{m} .
$$

The conventions are as in [7,8, 11] and the relation to section 2 is by $\left.f_{n}^{(r)}\right|_{\mu=0}=\bar{f}_{n}^{(r)} \sqrt{n}$.

As in flat space, (3.4) has the interpretation of continuity conditions on the vertex at the interaction, i.e. at $\tau=0$. The strategy, which we shall pursue (and which is in contrast to [11]) is to proceed as in flat space and stepwise solve for $f_{m}^{(3)}$, i.e., to find solutions $f_{m}^{(r)}$ to the set of equations

$$
\begin{aligned}
\sum_{p=1}^{\infty} f_{p}^{(3)} A_{p m}^{(r)} & =\frac{\alpha_{3}}{\alpha_{r}} f_{m}^{(r)} \\
\sum_{p=1}^{\infty} \sum_{r=1}^{3} \frac{1}{\alpha_{r}}\left(A^{(r)} U^{(r)}\right)_{m p} f_{p}^{(r)} & =-B_{m} .
\end{aligned}
$$

The Neumann matrices in the plane-wave case have been shown [24 to satisfy an analogous equation to (2.2),

$$
\bar{N}_{m n}^{r s}=-\frac{m n \alpha}{1-4 \mu \alpha K} \frac{\bar{N}_{m}^{r} \bar{N}_{n}^{s}}{\alpha_{s} \omega_{r m}+\alpha_{r} \omega_{s n}}
$$

where the Neumann vectors are

$$
\begin{aligned}
\bar{N}_{m}^{r} & =\left(\left(C^{-1} C_{r}\right)^{1 / 2} U_{r}^{-1} f^{(r)} \frac{1}{\alpha_{r}}\right)_{m} \\
& =\sqrt{\frac{\omega_{r m}}{m}} \frac{\left(\omega_{r m}+\mu \alpha_{r}\right)}{m} \frac{1}{\alpha_{r}} f_{m}^{(r)},
\end{aligned}
$$

and

$$
K=-\frac{1}{4} B^{t} \Gamma_{+}^{-1} B
$$

Further $C_{m n}=m \delta_{m n},\left(C_{r}\right)_{m n}=\omega_{r m} \delta_{m n}$ and the $f^{(r)}$ 's are defined as above.

Our strategy is now to apply the contour method to the sums in (3.6) and (3.7). From equation (3.6) one can again deduce the pole structure for $f^{(3)}(m)$. Assuming that the 
residues of the equations in (3.6) come from a single pole the conditions are that $f^{(3)}(m)$ has zeroes for $m \in-\mathbb{N}$ as well as $m(1+\beta) \in \mathbb{N}_{0}$ and has poles for $m \beta \in \mathbb{N}$. Thus, this fixes the pole and zero structure of the solution, however not the explicit functional dependence. The latter is determined by (3.7). For this, note the $\omega_{r p}$ term entering $U^{(r)}$. As discussed in [14], the integrals along the branch cuts that are present due to the square root will not contribute to the contour integral corresponding to the sum in question, if the $\mu$ and $p$ dependences are all packaged together into $\omega_{r p}$ and the integrand is odd in the imaginary part. Thus, in view of $(3.6)$, one is lead to the following explicit realization of the poles and zeroes in $f^{(3)}$

$$
f^{(3)}(m)=\tilde{f}_{m}^{(3)} \frac{\Gamma_{-\mu \beta}(-\beta m)}{\Gamma_{\mu(1+\beta)}(-(1+\beta) m) \Gamma_{\mu}(m)},
$$

where the particular choice of deformation parameters for the Gamma functions is chosen, in order to ensure that all branch cuts coincide. The function $\tilde{f}_{m}^{(3)}$ is determined much in the same way as we explained in detail for the flat-space discussion. Furthermore, one has to ensure that the all- $\mu$ solution reproduces the right flat-space limit. Note also, that the contour method requires that the integrand falls off suitably at infinity, so that the RHS of (2.12) vanishes. In the flat-space this discussion relied on applying the Stirling formula to the Gamma functions. For the plane-wave case, it will be relevant that the $\mu$-deformed Gamma-functions satisfy an analogous Stirling formula, which is proven in appendix B.

\subsection{Solutions to the vertex equations}

From the discussion in the last subsection, which in particular lead to the form (3.11), one obtains the following ansatz for the function $f^{(3)}(m)$, which has the correct flat-space limit. It further satisfies $\left.\frac{\partial f^{(3)}}{\partial \mu}\right|_{\mu=0}=\tau_{0} f^{(3)}$, which follows from equation (30) of [1] 0 . The ansatz reads

$$
f_{m}^{(3)}=\frac{m^{2}}{2 \omega_{m}} B_{m} y(1-y) e^{\tau_{0}\left(\mu-\omega_{m}\right)} \frac{\Gamma_{\mu}^{(1)}(m) \Gamma_{\mu}^{(2)}(m)}{\Gamma_{\mu}(m)} M\left(0^{+}\right)
$$

where we fixed as in 111 the gauge

$$
\alpha_{1}=y, \quad \alpha_{2}=1-y, \quad \alpha_{3}=-1
$$

5 Note this motivates the factor $1 / \omega_{m}$ as opposed to $1 /\left(\omega_{m}+\mu\right)$ which both have the correct flat space limit. 
and

$$
B_{m}=\frac{2}{\pi} \frac{\sin (m \pi(1-y))}{y(1-y) m^{3 / 2}}
$$

as well as

$$
M(z)=\frac{\Gamma_{\mu}(z) z}{\Gamma_{\mu y}(y z) y z \Gamma_{\mu(1-y)}((1-y) z)(1-y) z} .
$$

The factor $M\left(0^{+}\right)$is computed from (3.11) by imposing the equation (3.7) and thus is crucial in order to reproduce the correct residues. Note also that $M\left(0^{+}\right) \rightarrow 1$ as $\mu \rightarrow 0$. So, more explicitly we have

$$
\begin{aligned}
f^{(3)}(m) & =\frac{\sqrt{m}}{\pi} \sin (m \pi(1-y)) \frac{e^{\tau_{0}\left(\mu-\omega_{m}\right)}}{\omega_{m}} \frac{\Gamma_{\mu y}(y m) \Gamma_{\mu(1-y)}((1-y) m)}{\Gamma_{\mu}(m)} M\left(0^{+}\right) \\
& =-\frac{e^{\tau_{0}\left(\mu-\omega_{m}\right)}}{\omega_{m}} \frac{1}{\sqrt{m}(1-y)} \frac{\Gamma_{\mu y}(y m)}{\Gamma_{\mu(1-y)}(-(1-y) m) \Gamma_{\mu}(m)} M\left(0^{+}\right)
\end{aligned}
$$

Evaluation of the contour integrals corresponding to (3.6) for $r=1$ and $r=2$, which have only non-trivial residues at $m=-n / y$ and $m=-n /(1-y)$, respectively, results in

$$
\begin{aligned}
& f^{(1)}(n)=-\frac{(-1)^{n} e^{\tau_{0}\left(\mu+\omega_{\frac{n}{y}}\right)}}{\omega_{\frac{n}{y}} \sqrt{n}(1-y)} \frac{\Gamma_{\mu}\left(\frac{n}{y}\right)}{\Gamma_{\mu}^{(2)}\left(\frac{n}{y}\right) \Gamma_{\mu}^{(1)}\left(\frac{n}{y}\right)} M\left(0^{+}\right) \\
& f^{(2)}(n)=\frac{e^{\tau_{0}\left(\mu+\omega \frac{n}{1-y}\right)}}{\omega_{\frac{n}{1-y}} \sqrt{n} y} \frac{\Gamma_{\mu}\left(\frac{n}{1-y}\right)}{\Gamma_{\mu}^{(1)}\left(\frac{n}{1-y}\right) \Gamma_{\mu}^{(2)}\left(\frac{n}{1-y}\right)} M\left(0^{+}\right) .
\end{aligned}
$$

Note that in evaluating the contour integrals for each value for $r$ the branch cuts coincide, the integrand being odd along the cuts, which is of course crucial for the applicability of the contour method. These solutions can be put into the closed form

$$
f_{m}^{(r)}=\frac{e^{\tau_{0}\left(\mu+\omega \frac{m}{\alpha_{r}}\right)}}{\sqrt{m}\left(-\alpha_{r}-\alpha_{r+1}\right) \omega_{\frac{m}{\alpha_{r}}}} \frac{\Gamma_{\mu}^{(r+1)}\left(-\frac{m}{\alpha_{r}}\right)}{\Gamma_{\mu}^{(r)}\left(\frac{m}{\alpha_{r}}\right) \Gamma_{\mu}^{(r-1)}\left(\frac{m}{\alpha_{r}}\right)} M\left(0^{+}\right)
$$

which beautifully generalizes the corresponding expression in flat-space (2.31) taking into account the difference in conventions.

Next (3.7) needs to be checked. Thus consider the following contour integral, which corresponds to the sum of the $r=1$ term in (3.7) ( $c f$. flat space analysis)

$$
-M\left(0^{+}\right) \oint \frac{d n}{2 \pi i} \frac{2 y(-1)^{m+1} \sqrt{m} \sin (m \pi y)}{n \sin (\pi n)} \frac{e^{\tau_{0}\left(\mu+\omega_{\frac{n}{y}}\right)}}{n^{2}-m^{2} y^{2}} \frac{\left(\omega_{\frac{n}{y}}-\mu\right)}{\omega_{\frac{n}{y}}} \frac{\Gamma_{\mu(1-y)}\left(-\frac{n}{y}(1-y)\right)}{\Gamma_{\mu}\left(-\frac{n}{y}\right) \Gamma_{\mu y}(n)} .
$$


The residues at $n \in \mathbb{N}$ give the $r=1$ term, the residues at $n(1-y) / y \in \mathbb{N}$ give the $r=2$ term and the residue at $n=-m y$ gives the $r=3$ term in (3.7). We are left only with the integral around the branch cut, which runs from $n=i y \mu$ to $n=-i y \mu$. Since the integrand is odd along either side of the cut the line integrals vanish - however the integrand is actually singular at $n=0$ and thus we are left with two semicircular contours on either side of the branch cut, which we will call $C_{+}$and $C_{-}$, see figure 1 . One might be tempted to think this contribution is just the residue of the integrand at $n=0$ however things are not quite as simple as this, since the integrand is not defined at this point with our choice of branch cut. What we can do though is take the residue of the factors not depending on $\omega_{n}$ and then on the right side (i.e., along $C_{+}$) take the limit $n \rightarrow 0^{+}$of the rest and on the left take the limit $n \rightarrow 0^{-}$.

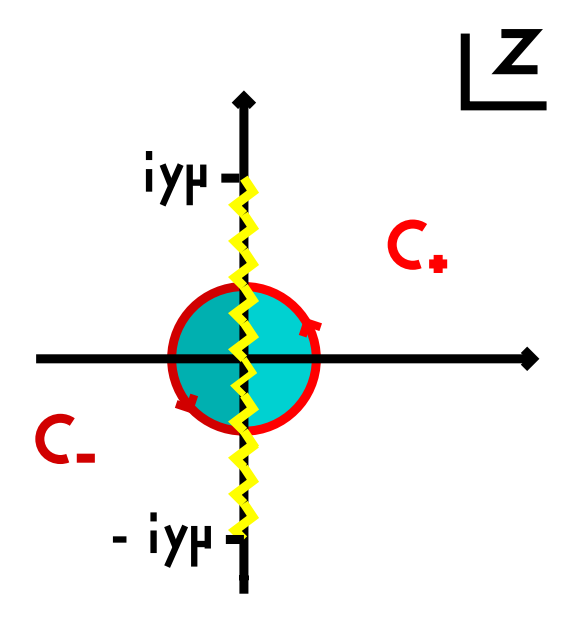

Figure 1 The contours $C_{ \pm}$.

In the present case, the only contribution comes from $C_{-}$(note the integrand is not regular at the branch points, however the integral around them still vanishes, in fact goes as $O\left(\epsilon^{1 / 2}\right)$ where $\epsilon$ is the radius of the contour around a branch point, see [14]). We find that the contribution from $C_{-}$is

$$
-\frac{2(-1)^{m} \sin m \pi y}{\pi m^{3 / 2} y(1-y)} M\left(0^{+}\right) M\left(0^{-}\right) .
$$

It is easy to verify that $M\left(0^{+}\right) M\left(0^{-}\right)=1$, which leaves the contribution from $C_{-}$to be exactly $B_{m}$ thus completing the proof.

Finally, we need to compute the quantity $K$, which is defined by

$$
K=-\frac{1}{4} B^{t} \Gamma_{+}^{-1} B=-\frac{1}{4} \sum_{n=1}^{\infty} B_{n} f_{n}^{(3)} .
$$


For the computation, we need to consider the contour integral

$$
-\frac{2 M\left(0^{+}\right)}{y(1-y)} \oint \frac{d m}{2 \pi i} \frac{M(-m) e^{\tau_{0}\left(-\omega_{m}+\mu\right)}}{m \omega_{m}} .
$$

The residues at $-m \in \mathbb{N}$ give $-4 K$ and since there are no other poles we are left with the integrals around the branch cut. There is a singularity in the integrand at $m=0$ which on both sides looks like a simple pole. Computing we get

$$
-\frac{2 M\left(0^{+}\right)}{y(1-y)} \int_{C_{+}} \frac{d m}{2 \pi i} \frac{M(-m) e^{\tau_{0}\left(-\omega_{m}+\mu\right)}}{m \omega_{m}}=-\frac{M\left(0^{+}\right) M\left(0^{-}\right)}{\mu y(1-y)},
$$

and

$$
-\frac{2 M\left(0^{+}\right)}{y(1-y)} \int_{C-} \frac{d m}{2 \pi i} \frac{M(-m) e^{\tau_{0}\left(-\omega_{m}+\mu\right)}}{m \omega_{m}}=\frac{M\left(0^{+}\right)^{2} e^{2 \tau_{0} \mu}}{\mu y(1-y)} .
$$

Therefore we find that

$$
K=\frac{1}{4 y(1-y) \mu}\left(M\left(0^{+}\right)^{2} e^{2 \mu \tau_{0}}-1\right)
$$

which we see has the correct flat space limit, $K(\mu=0)=\tau_{0} /(2 y(1-y))$.

We should emphasize that in this application of the contour method of [14] it has been crucial that the circular integrals around the branch points, as well as the integrals at infinity vanish - the latter was shown by applying the generalization of Stirling's formula of appendix B.

\subsection{Comments}

A couple of remarks are in order, in particular in view of the comparison with the solution obtained in [11]. First recall that the solution $Y_{m}(\mu, y)$ in [11] is to be compared with our solution $f_{m}^{(3)}$. In this comparison, one will observe that the solution in eq. (52) of [11] only agrees with (3.12), if $\alpha_{3} \rightarrow-\alpha_{3}$ as well as the sign of the $r=3$ term in the exponent is flipped, i.e., if

$$
Y_{m}(\mu, y)=\exp \left[\left(\mu-\omega_{m}\right) \tau_{0}+\sum_{r=1}^{2}\left(\phi_{r}-\phi_{m r}\right)-\left(\phi_{3}-\phi_{m 3}\right)\right] \frac{m}{2 \omega_{m}} B_{m}
$$

with $\alpha_{3}$ replaced by 1 . Note that the solution as it stands in [11] would be divergent for integral $m$, and thus seems inconsistent 6 .

6 The authors of [11] have confirmed the sign discrepancies in the equation in question. We would like to thank Y. He and M. Spradlin for discussions on this point. 


\section{Large- $\mu$ asymptotics}

The large- $\mu$ asymptotics of expressions in plane-wave string theory are of the foremost interest in the context of the BMN correspondence, as these are to be compared with perturbative (in $\lambda^{\prime}$ ) gauge theory data. Given our expressions for the Neumann matrices, the only non-trivial input in determining these asymptotic expressions are the large- $\mu$ asymptotics of the $\mu$-deformed Gamma-functions, which we derive in appendix C. In applying these one has to keep in mind that the asymptotic formulae only apply to $\Gamma^{(r)}(z)$ for $|\arg (z)|<\pi$, thus before applying the asymptotics to the functions $f^{(r)}(m)$ one has to ensure that upon suitable application of the reflection identities this condition is satisfied for the arguments.

First note that $M\left(0^{+}\right)$has the asymptotics, using the $\Gamma$-function asymptotics of the appendix $\mathrm{C}$, given by $\mathrm{G}$

$$
M\left(0^{+}\right) \approx e^{-\tau_{0} \mu} \sqrt{\frac{1}{4 \pi \mu y(1-y)}} .
$$

Applying these asymptotics upon (3.18) and (3.19), we obtain

$$
\begin{aligned}
& f_{n}^{(1)} \approx \frac{(-1)^{n}}{2 \pi \sqrt{2 \mu} y(1-y)} \frac{\sqrt{n}}{\omega_{\frac{n}{y}} \sqrt{\omega_{\frac{n}{y}}+\mu}} \\
& f_{n}^{(2)} \approx \frac{1}{2 \pi \sqrt{2 \mu} y(1-y)} \frac{\sqrt{n}}{\omega_{\frac{n}{(1-y)}} \sqrt{\omega_{\frac{n}{(1-y)}}+\mu}} .
\end{aligned}
$$

For $f^{(3)}(n)$, the asymptotic formula is only applicable to 3.16 , resulting in

$$
f_{m}^{(3)} \approx \frac{1}{\pi} \sin \left(m \pi \alpha_{2}\right) \frac{\alpha_{3}}{\sqrt{m}} \frac{1}{\sqrt{2 \mu} \alpha} \frac{\sqrt{\omega_{-\frac{m}{\alpha_{3}}}+\mu}}{\omega_{\frac{m}{\alpha_{3}}}} .
$$

This expansion agrees precisely with [11]. The Neumann vectors

$$
\begin{aligned}
\bar{N}_{m}^{r} & =\sqrt{\frac{\omega_{r m}}{m}} \frac{\left(\omega_{r m}+\mu \alpha_{r}\right)}{m} f_{m}^{(r)} \frac{1}{\alpha_{r}} \\
& =\sqrt{\frac{\omega_{r m}}{m}} \frac{m}{\left(\omega_{r m}-\mu \alpha_{r}\right)} f_{m}^{(r)} \frac{1}{\alpha_{r}}
\end{aligned}
$$

thus have the asymptotic behaviour for $r=1,2$

$$
\bar{N}_{m}^{r} \approx-\frac{(-1)^{r(n+1)} \alpha_{r}^{1 / 2}}{2 \pi \alpha \sqrt{2 \mu} m} \frac{\sqrt{\left(\omega_{r m}+\mu \alpha_{r}\right)}}{\sqrt{\omega_{r m}}}
$$

7 As in 11], $A(\mu) \approx B(\mu)$ means $A(\mu)=B(\mu)+O\left(e^{-\left|\alpha_{r}\right| \mu}\right)$. 
as well as for $r=3$

$$
\bar{N}_{m}^{3} \approx-\frac{1}{\pi} \sin \left(m \pi \alpha_{2}\right) \frac{1}{\sqrt{2 \mu} \alpha} \sqrt{\frac{\left|\alpha_{3}\right|}{\omega_{3 m}\left(\omega_{3 m}-\mu \alpha_{3}\right)}} .
$$

Finally we may study the large- $\mu$ asymptotics of $K$. Inspecting (3.27) we see that we only need (4.1), which leads to

$$
K \approx-\frac{1}{4 y(1-y) \mu}+\frac{1}{16 \pi y^{2}(1-y)^{2} \mu^{2}}
$$

These agree with the findings of [11].

\subsection{Exponential corrections}

In this section we will concentrate on explicitly extracting the first exponential correction to the Neumann vectors we have derived, thus going further than the results given in [11]. These corrections could have interesting implications for the gauge theory.

The problem reduces to finding the large- $\mu$ exponential corrections to the deformed Gamma-functions. Here we give a brief argument and one can find a more rigorous derivation in appendix D. In appendix D we show the following key result

$$
\frac{\partial}{\partial \mu} \log \Gamma_{\mu}^{(r)}(z)_{\exp }=-\frac{\sqrt{\left|\alpha_{r}\right| \mu}}{\omega_{z}} e^{-2 \pi\left|\alpha_{r}\right| \mu}\left[1+O\left(\frac{1}{\mu}\right)\right] .
$$

If one does an integration with respect to $\mu$ by parts (i.e., integrate the $\left.e^{-2 \pi\left|\alpha_{r}\right| \mu}\right)$, then we can prove that

$$
\log \Gamma_{\mu}^{(r)}(z)_{\exp }=\frac{\sqrt{\left|\alpha_{r}\right| \mu}}{2 \pi\left|\alpha_{r}\right| \omega_{z}} e^{-2 \pi\left|\alpha_{r}\right| \mu}\left[1+O\left(\frac{1}{\mu}\right)\right],
$$

the details of which are provided in appendix D. This essentially means that the remaining integral simply contributes to the $O(1 / \mu)$ part. Now it is a simple matter of some algebra to deduce the corrections to the Neumann vectors. We find the following result

$$
\begin{aligned}
\frac{\Gamma_{\mu}^{(1)}(z) \Gamma_{\mu}^{(2)}(z)}{\Gamma_{\mu}(z)} & =\frac{\sqrt{2 \pi} e^{\omega_{z} \tau_{0}}}{\sqrt{y(1-y)}} \frac{\sqrt{\omega_{z}+\mu}}{z}\left\{1+\frac{\sqrt{\mu}}{2 \pi \omega_{z}}\left(\frac{e^{-2 \pi y \mu}}{\sqrt{y}}\left[1+O\left(\frac{1}{\mu}\right)\right]\right.\right. \\
& \left.\left.+\frac{e^{-2 \pi(1-y) \mu}}{\sqrt{1-y}}\left[1+O\left(\frac{1}{\mu}\right)\right]-e^{-2 \pi \mu}\left[1+O\left(\frac{1}{\mu}\right)\right]\right)\right\} .
\end{aligned}
$$

Using this it is straightforward to deduce the corrections to $f_{m}^{(r)}$. 
Curiously, it can be shown that the exponential corrections are related to the Casimir energy of a two-dimensional massive boson on a cylindrical world-sheet with periodic boundary condition. The Casimir energy is given by [25,26]

$$
\Delta_{\mu \alpha_{r}}=-\frac{1}{(2 \pi)^{2}} \sum_{p=1}^{\infty} \int_{0}^{\infty} d t e^{-p^{2} t-\pi^{2} \alpha_{r}^{2} \mu^{2} / t}
$$

using which it can be easily shown that the exponential corrections for the deformed Gamma-function satisfies the following relation

$$
\frac{\partial \log \Gamma_{\mu}^{(r)}(z)_{\exp }}{\partial \mu}=\frac{1}{\omega_{z} \alpha_{r}} \frac{\partial \Delta_{\mu \alpha_{r}}}{\partial \mu}=\frac{1}{\omega_{z} \mu} \frac{\partial \Delta_{\mu \alpha_{r}}}{\partial \alpha_{r}}
$$

$\alpha$ parametrizes the length of the world-sheet. Since the $\alpha$-derivative of the energy can be interpreted as pressure, these corrections probably arise due to the tidal forces in this background 25]. The exact physical significance of this result, particularly on the gauge theory side needs to be explored.

It is tempting to extend the analysis applied for the first term in (4.9) in order to extract the full series of exponential corrections. In this paper, we shall content ourselves by giving a conjectural expression for the series (4.9),

$$
\log \Gamma_{\mu}^{(r)}(z)_{\exp }=\sum_{n=1}^{\infty} e^{-2 \pi n\left|\alpha_{r}\right| \mu} \frac{\sqrt{\left|\alpha_{r}\right| \mu}}{2 \pi\left|\alpha_{r}\right| \sqrt{n} \omega_{z}}\left(1+O\left(\frac{1}{\mu}\right)\right)
$$

where the conjecture is, that the $O(1 / \mu)$ terms do not lead to any mixing of the exponentials in the series. We provide some arguments in favour of this conjecture in appendix D.1.

Using the above conjecture and upon expanding the exponential, in general the exponential corrections can be written schematically as

$$
\sum_{n=1}^{\infty} g_{n}\left(\mu, \alpha_{r}\right) e^{-2 \pi n \mu\left|\alpha_{r}\right|}
$$

which suggests the interpretation of these quantities in terms of a series of non-perturbative corrections. Note, that in deriving the exponential corrections we made use of the asymptotic expansion of the Bessel function $K_{\nu}(x)$. It is well known that this expansion is not Borel summable. The exact significance of this for the dual gauge theory needs to be understood. 


\section{Plane-wave Neumann matrix manual}

The purpose of this section is to summarise our main results, in order to facilitate the comparison with gauge theory calculations for which the Neumann matrices are essential. We give the expressions for the Neumann matrices valid for all $\mu$, the large- $\mu$ expansion as well as the first exponential corrections, which we have determined explicitly. The subsequent exponential terms are only conjectural and can be obtained from (4.13).

So, to summarize, we have expressed the plane-wave Neumann matrices as

$$
\bar{N}_{m n}^{r s}=-\frac{m n \alpha}{1-4 \mu \alpha K} \frac{\bar{N}_{m}^{r} \bar{N}_{n}^{s}}{\alpha_{s} \omega_{r m}+\alpha_{r} \omega_{s n}},
$$

where the Neumann vectors $\bar{N}_{m}^{r}$ are related to the functions $f_{m}^{(r)}$ via

$$
\bar{N}_{m}^{r}=\sqrt{\frac{\omega_{r m}}{m}} \frac{\left(\omega_{r m}+\mu \alpha_{r}\right)}{m} \frac{1}{\alpha_{r}} f_{m}^{(r)} .
$$

We have determined the explicit form of these functions in (3.20), and furthermore $K$ was computed in (3.27). Putting all this together, we obtain the Neumann matrices for all $\mu$-values to be

$$
\begin{aligned}
\bar{N}_{m n}^{r s}= & -\frac{4}{m n} e^{\tau_{0}\left(\omega \frac{m}{\alpha_{r}}+\omega \frac{n}{\alpha_{s}}\right)_{\times}} \\
& \times \frac{\alpha}{\left(\alpha_{r}+\alpha_{r+1}\right)\left(\alpha_{s}+\alpha_{s+1}\right)} \frac{\left(\omega_{r m}+\mu \alpha_{r}\right)\left(\omega_{s n}+\mu \alpha_{s}\right)}{\left(\omega_{r m} \omega_{s n}\right)^{1 / 2}\left(\alpha_{s} \omega_{r m}+\alpha_{r} \omega_{s n}\right)} \times \\
& \times\left(\frac{\Gamma_{\mu}^{(r+1)}\left(-\frac{m}{\alpha_{r}}\right)}{\Gamma_{\mu}^{(r)}\left(\frac{m}{\alpha_{r}}\right) \Gamma_{\mu}^{(r-1)}\left(\frac{m}{\alpha_{r}}\right)}\right)\left(\frac{\Gamma_{\mu}^{(s+1)}\left(-\frac{n}{\alpha_{s}}\right)}{\Gamma_{\mu}^{(s)}\left(\frac{n}{\alpha_{s}}\right) \Gamma_{\mu}^{(s-1)}\left(\frac{n}{\alpha_{s}}\right)}\right),
\end{aligned}
$$

where the $\mu$-deformed Gamma-functions are defined in (3.1) and (3.2). Applying the large$\mu$ asymptotics for the deformed Gamma-functions obtained in appendix $\mathrm{C}$ one can extract the large- $\mu$ behavior, which is of interest for comparison with the gauge theory. We have given the explicit forms of the large- $\mu$ Neumann vectors in section 4 , and the asymptotics for (5.1) are straight-forwardly obtained from (4.5), (4.6) and (4.7).

The first term in the exponential corrections to these large- $\mu$ asymptotics, which had so far not been determined, follow from the exponential corrections of the Gammafunctions in (4.10), together with (5.1). 


\section{Conclusions}

In this paper we have derived the bosonic Neumann matrices in plane-wave string theory using the contour method developed in [14], which allows to express the result in terms of $\mu$-deformed Gamma-functions. This approach not only simplifies the derivation of the Neumann matrices and their large- $\mu$-asymptotics, the latter being in agreement with [11], but allows to extract exponential corrections, i.e., terms of $O\left(e^{-2 \pi\left|\alpha_{r}\right| \mu}\right)$. We have derived an explicit form for the first term in these exponential corrections, and provided a conjectural formula for the leading order in $1 / \mu$ terms in the full exponential series. A few open questions and remarks are in order.

- The dual gauge-theoretical interpretation of the exponential corrections has certainly so far been elusive. The explicit form for the first term in the exponential corrections, which we derived, provides some explicit quantity that could be compared to the gauge theory. In terms of the effective 't Hooft coupling, $\lambda^{\prime}=1 /\left(\mu p^{+} \alpha^{\prime}\right)^{2}$, the result is proportional to $e^{-2 \pi / \sqrt{\lambda^{\prime}}}$. In particular, these should correspond to non-perturbative effects, which are remotely reminiscent of contributions that arise from D-branes in string theory. A vital question that hereby arises is then: What objects on the gauge theory side could be attributed such corrections?

- The exponential corrections were shown to be related to the Casimir energy of a massive two-dimensional boson on a cylinder. Is this merely a mathematical coincidence or can it be attributed more physical significance?

- It would be very interesting to use the results obtained in the present paper as well as in [14] in order to compute scattering of closed and open strings. In particular, the open string cubic vertex is of course closely related to the closed cubic vertex and could be used in order to compute scattering of open strings in orientifold theories or with D-branes, such as the ones constructed in [25,27,28,29]. Furthermore, these should be compared to gauge theoretical computations including operators dual to D-branes, such as in [30, 31, 32].

- As a mathematical curiosity, it is conceivable that the contour method could be used more generally to derive integral transforms, in the same way that the present paper gives a systematic way to obtain the integral transform used in [1] to derive the cubic vertex.

- Finally, it is tempting to conjecture that all flat space amplitudes, which can be expressed in terms of Gamma-functions, can be carried over in the plane-wave background by replacing them with suitable $\mu$-deformed Gamma-functions. 


\section{Acknowledgments}

We thank Michael B. Green, Sean Hartnoll, Yang-Hui He, Minxin Huang, Stefano Kovacs, S. Prem Kumar and Mark Spradlin for very useful discussions. JL is supported by EPSRC. SSN thanks the University of Pennsylvania and the IAS, Princeton, for hospitality during the final stages of this work. AS acknowledges financial support from the Gates Cambridge Trust and the Matthews' scholarship of Gonville and Caius College, Cambridge.

\section{Appendix A. Notations and Conventions}

The following definitions have been used in the main body of the paper

$$
\begin{aligned}
A_{m n}^{(1)} & =\frac{2}{\pi}(-1)^{m+n+1} \sqrt{m n} \frac{\beta \sin (m \pi \beta)}{n^{2}-m^{2} \beta^{2}} \\
A_{m n}^{(2)} & =\frac{2}{\pi}(-1)^{m+1} \sqrt{m n} \frac{(\beta+1) \sin (m \pi \beta)}{n^{2}-m^{2}(\beta+1)^{2}} \\
A_{m n}^{(3)} & =\delta_{m n} \\
B_{m} & =\frac{2}{\pi} \frac{\alpha_{3}}{\alpha_{1} \alpha_{2}}(-1)^{m+1} \frac{\sin (m \pi \beta)}{m^{3 / 2}}
\end{aligned}
$$

which arise in the Fourier mode expansion of the vertex equation. Further it is useful to define

$$
\begin{aligned}
C_{m n} & =m \delta_{m n} \\
\left(C_{r}\right)_{m n} & =\omega_{r m} \delta_{m n} \\
\left(U^{(r)}\right)_{m n} & =\delta_{m n} \frac{\left(\omega_{r m}-\alpha_{r} \mu\right)}{m} .
\end{aligned}
$$

Here, $\beta=\alpha_{1} / \alpha_{3}$. We will mostly work in with the choice $\alpha_{1}=y$ and $\alpha_{2}=1-y$ and hence $\alpha_{3}=-1$, as in [11].

\section{Appendix B. Generalisation of Stirling's formula}

In this section we analyse the large $z$ asymptotics of the Gamma function $\Gamma_{\mu}(z)$. Recall this is defined as [14]

$$
\Gamma_{\mu}(z)=\frac{e^{-\gamma \omega_{z}}}{z} \prod_{n=1}^{\infty} \frac{n}{\omega_{z}+\omega_{n}} e^{\omega_{z} / n}
$$


Using the Weierstrass definition of $\Gamma(z)$ implies

$$
\log \left(\frac{\Gamma_{\mu}(z)}{\Gamma(z)}\right)=-\gamma\left(\omega_{z}-z\right)+\sum_{n=1}^{\infty} \log \left(\frac{z+n}{\omega_{z}+\omega_{n}}\right)+\frac{\omega_{z}-z}{n}
$$

Note that $\lim _{z \rightarrow \infty}\left(\omega_{z}-z\right)=0$. This allows us to deduce that

$$
\lim _{z \rightarrow \infty} \log \left(\frac{\Gamma_{\mu}(z)}{\Gamma(z)}\right)=0
$$

and therefore that

$$
\Gamma_{\mu}(z) \sim \Gamma(z) \sim \sqrt{2 \pi} z^{z-1 / 2} e^{-z}
$$

as $z \rightarrow \infty$ and of course for $|\arg z|<\pi$.

\section{Appendix C. Large- $\mu$ asymptotics of the deformed Gamma-functions}

The large- $\mu$ asymptotics are derived in a similar fashion as in the appendices of [14], applying various techniques of [33]. Taking the log of both sides of (3.1) and differentiating with respect to $\mu$ leads to

$$
\frac{\partial}{\partial \mu} \log \Gamma_{\mu}^{(r)}(z)=\frac{\alpha_{r} \mu}{\omega_{z}}\left[\sum_{n=1}^{\infty}\left(\frac{1}{n}-\frac{1}{\omega_{r n}}\right)-\gamma\right] .
$$

So we need to consider the asymptotics of

$$
S=\sum_{n=1}^{\infty}\left(\frac{1}{n}-\frac{1}{\omega_{r n}}\right)
$$

Differentiating both sides with respect to $\mu$ implies

$$
\frac{\partial S}{\partial \mu}=\sum_{n=1}^{\infty} \frac{\alpha_{r}^{2} \mu}{\omega_{r n}^{3}}
$$

Using the results in the appendix of [14]

$$
\sum_{n=1}^{\infty} \frac{1}{\omega_{r n}^{3}}=-\frac{1}{2\left(\alpha_{r} \mu\right)^{3}}+\frac{1}{\left(\alpha_{r} \mu\right)^{2}}+O\left(e^{-\left|\alpha_{r}\right| \mu}\right),
$$

which after integrating leads to

$$
S=\frac{1}{2 \mu \alpha_{r}}+\log \mu+c\left(\alpha_{r}\right)+O\left(e^{-\left|\alpha_{r}\right| \mu}\right)
$$


where $c\left(\alpha_{r}\right)$ is a constant of integration. Differentiating with respect to $\alpha_{r}$ leads to

$$
\frac{\partial S}{\partial \alpha_{r}}=-\frac{1}{2 \mu \alpha_{r}^{2}}+\frac{\partial c}{\partial \alpha_{r}}+O\left(e^{-\left|\alpha_{r}\right| \mu}\right) .
$$

One should now differentiate $S$ with respect to $\alpha_{r}$ and then take the large- $\mu$ limit of the resulting expression to compare with this one. This leads to

$$
\frac{\partial S}{\partial \alpha_{r}}=\sum_{n=1}^{\infty}\left(\frac{\mu^{2} \alpha_{r}}{\omega_{r n}^{3}}\right)=-\frac{1}{2 \alpha_{r}^{2} \mu}+\frac{1}{\alpha_{r}}+O\left(e^{-\left|\alpha_{r}\right| \mu}\right) .
$$

Now comparing with equation (C.6) we get

$$
\frac{\partial c}{\partial \alpha_{r}}=\frac{1}{\alpha_{r}}
$$

implying that

$$
c\left(\alpha_{r}\right)=\log \alpha_{r}+c,
$$

and therefore

$$
S=\frac{1}{2 \mu \alpha_{r}}+\log \left(\mu \alpha_{r}\right)+c+O\left(e^{-\left|\alpha_{r}\right| \mu}\right) .
$$

Substituting this into (C.1) and then integrating with respect to $\mu$ we arrive at

$$
\begin{aligned}
\log \Gamma_{\mu}^{(r)}(z) & =\alpha_{r} \omega_{z}\left(c-\gamma-1+\log \left(\mu \alpha_{r}\right)\right)+\frac{1}{2} \log \left(\omega_{z}+\mu\right) \\
& +z \alpha_{r} \log \left(\frac{\omega_{z}+z}{\mu}\right)+K\left(z, \alpha_{r}\right)+O\left(e^{-\left|\alpha_{r}\right| \mu}\right),
\end{aligned}
$$

where $K\left(z, \alpha_{r}\right)$ comes from integrating with respect to $\mu$. This function can be determined by taking the large- $\mu$ asymptotics of $\frac{\partial}{\partial z} \log \Gamma_{\mu}^{(r)}(z)$ and comparing to the $z$ and $\alpha_{r}$ derivatives of ([C.11), which we will do next. Taking the partial derivative with respect to $z$ of (C.11) leads to

$\frac{\partial}{\partial z} \log \Gamma_{\mu}^{(r)}(z) \approx \frac{z}{2\left(\mu+\omega_{z}\right) \omega_{z}}+\left(c-\gamma-1+\log \alpha_{r} \mu\right) \frac{\alpha_{r} z}{\omega_{z}}+\alpha_{r} \log \left(\frac{z+\omega_{z}}{\mu}\right)+\frac{z \alpha_{r}}{\omega_{z}}+\frac{\partial K}{\partial z}$,

while the $z$ logarithmic derivative of (3.1) leads to

$$
-\gamma \alpha_{r} \frac{z}{\omega_{z}}-\frac{1}{z}-\frac{\alpha_{r} z}{\omega_{z}} \sum_{n=1}^{\infty}\left(\frac{1}{\omega_{r n}+\alpha_{r} \omega_{z}}-\frac{1}{n}\right) .
$$


Taking the limit $\mu \rightarrow \infty$ in both equations results in the condition

$$
\frac{\partial K}{\partial z}=-\frac{1}{z}
$$

Now taking the partial derivative with respect to $\alpha_{r}$ of (C.11) one obtains

$$
\frac{\partial}{\partial \alpha_{r}} \log \Gamma_{\mu}^{(r)}(z) \approx\left(c-\gamma+\log \left(\alpha_{r} \mu\right)\right) \omega_{z}+z \log \left(\frac{z+\omega_{z}}{\mu}\right)+\frac{\partial K}{\partial \alpha_{r}}
$$

while the $\alpha_{r}$ logarithmic derivative of (3.1) implies

$$
-\gamma \omega_{z}-\frac{1}{\alpha_{r}}-\sum_{n=1}^{\infty}\left(\frac{1}{\omega_{r n}+\alpha_{r} \omega_{z}}\left(\frac{\alpha_{r} \mu^{2}}{\omega_{r n}}+\omega_{z}\right)-\frac{\omega_{z}}{n}\right) .
$$

Comparing the above two equations at $z=0$ and using (C.10) leads to the condition

$$
\frac{\partial K}{\partial \alpha_{r}}=-\frac{1}{2 \alpha_{r}}
$$

Therefore we conclude that

$$
K\left(z, \alpha_{r}\right)=-\log z-\frac{1}{2} \log \alpha_{r}+c^{\prime}
$$

Finally we have the desired asymptotic expression for the deformed Gamma-functions for $|\arg z|<\pi$

$$
\begin{aligned}
\log \Gamma_{\mu}^{(r)}(z) & =\alpha_{r} \omega_{z}\left(c-\gamma-1+\log \left(\mu \alpha_{r}\right)\right)+\frac{1}{2} \log \left(\alpha_{r} \omega_{z}+\alpha_{r} \mu\right) \\
& +z \alpha_{r} \log \left(\frac{\omega_{z}+z}{\mu}\right)-\log \left(z \alpha_{r}\right)+c^{\prime}+O\left(e^{-\left|\alpha_{r}\right| \mu}\right) .
\end{aligned}
$$

The constants $c$ and $c^{\prime}$ can actually be determined, and we will in fact need $c^{\prime}$ explicitly. To determine these constants we employ the large $z$-asymptotics formula derived in appendix B which is valid for all $\mu$. For large $z$, the RHS of (C.19) (ignoring the $O\left(e^{-\left|\alpha_{r}\right| \mu}\right.$ ) contribution) is asymptotic to

$$
\left(\alpha_{r} z-\frac{1}{2}\right) \log \left(\alpha_{r} z\right)+\alpha_{r} z(c-\gamma-1+\log 2)+c^{\prime}
$$

Comparing this to Stirling's formula (which is valid for all $\mu$ ) we see that we must have $c=\gamma-\log 2$ and $c^{\prime}=\log \sqrt{2 \pi}$. 


\section{Appendix D. $O\left(e^{-\mu}\right)$ corrections}

We shall now derive the exponential corrections in the large- $\mu$ expansion of the deformed Gamma-functions. In equation (C.3), the $O\left(e^{-\mu}\right)$ term is given by

$$
\frac{\partial S_{e}}{\partial \mu}=2 \alpha_{r}^{2} \mu \int_{0}^{\infty} \frac{d s}{\mu^{2} \alpha_{r}^{2}} e^{-s} \sum_{n=1}^{\infty} e^{-\frac{n^{2} \pi^{2} \mu^{2} \alpha_{r}^{2}}{s}}
$$

which can be written in terms of the modified Bessel function of the second kind as

$$
4 \pi\left|\alpha_{r}\right| \sum_{n=1}^{\infty} n K_{1}\left(2 n \pi \mu\left|\alpha_{r}\right|\right)
$$

where we have used the integral represenation $K_{1}(x)=\frac{1}{x} \int_{0}^{\infty} d t e^{-t-x^{2} / 4 t}$. Integrating with respect to $\mu$ leads to

$$
S_{e}=-2 \sum_{n=1}^{\infty} K_{0}\left(2\left|\alpha_{r}\right| \mu n \pi\right) .
$$

Thus the $O\left(e^{-\mu}\right)$ terms in (C.1) are given by

$$
F(z, \mu) \equiv-2 \sum_{n=1}^{\infty} \frac{\alpha_{r} \mu}{\omega_{z}} K_{0}\left(2\left|\alpha_{r}\right| \mu n \pi\right) .
$$

The large- $\mu$ behaviour of this quantity is readily deduced from that of the Kelvin functions 8 , which gives

$$
F(z, \mu)=-\frac{\sqrt{\left|\alpha_{r}\right| \mu}}{\omega_{z}} e^{-2 \pi\left|\alpha_{r}\right| \mu}\left[1+O\left(\frac{1}{\mu}\right)\right] .
$$

Thus we are left with evaluating $\int F(z, \mu) d \mu$. We now prove the following formula crucial for the evaluation of the above integral

$$
I(z)=\int_{\mu}^{\infty} d t \frac{\sqrt{t}}{\sqrt{t^{2}+z^{2}}} e^{-\xi t}=\frac{\sqrt{\mu} e^{-\xi \mu}}{\xi \omega_{z}}\left[1+O\left(\frac{1}{\mu}\right)\right] .
$$

We will prove the formula for real $z$ as this is all we will need. The argument is elementary and goes as follows. Integrate by parts to give

$$
I(z)=\frac{\sqrt{\mu} e^{-\xi \mu}}{\xi \omega_{z}}-\frac{1}{2 \xi} \int_{\mu}^{\infty} d t e^{-\xi t} \frac{\left(z^{2}-t^{2}\right)}{\sqrt{t}\left(z^{2}+t^{2}\right)^{3 / 2}} .
$$

8 Note $K_{\nu}(x)=\sqrt{\frac{\pi}{2 x}} e^{-x}(1+O(1 / x))$. 
Since $\left|z^{2}-t^{2}\right|<z^{2}+t^{2}$ for real $z$, we have

$$
\left|\frac{\left(z^{2}-t^{2}\right)}{\sqrt{t}\left(z^{2}+t^{2}\right)^{3 / 2}}\right|<\frac{1}{\sqrt{t}\left(z^{2}+t^{2}\right)^{1 / 2}} \leq \frac{1}{\sqrt{\mu}\left(z^{2}+\mu^{2}\right)^{1 / 2}}
$$

wherefore

$$
\left|\frac{1}{2 \xi} \int_{\mu}^{\infty} d t e^{-\xi t} \frac{\left(z^{2}-t^{2}\right)}{\sqrt{t}\left(z^{2}+t^{2}\right)^{3 / 2}}\right|<\frac{e^{-\xi \mu}}{2 \xi^{2} \sqrt{\mu} \omega_{z}} .
$$

Hence we have proven (D.6). Note we have not restricted $\mu$ in this proof at all. Thus one might expect to extend this to complex $z$ for large- $\mu$. Using this we conclude that

$$
\int_{\mu}^{\infty} F(z, \mu) d \mu=\frac{\sqrt{\left|\alpha_{r}\right| \mu}}{2 \pi\left|\alpha_{r}\right| \omega_{z}} e^{-2 \pi\left|\alpha_{r}\right| \mu}\left[1+O\left(\frac{1}{\mu}\right)\right] .
$$

Finally we comment on the connection to [11]. For this, we make use of the following integral representation

$$
K_{0}(x \mu)=\int_{0}^{\infty} d t \frac{e^{-x \sqrt{t^{2}+\mu^{2}}}}{\sqrt{t^{2}+\mu^{2}}}
$$

valid for $x>0$, see [34, to express the whole of the exponential corrections in a different form. Using this we may sum $S_{e}$, given by (D.3), which implies

$$
S_{e}=-2 \int_{0}^{\infty} d t \frac{1}{\sqrt{t^{2}+\mu^{2}}\left[e^{2 \pi \alpha_{r} \sqrt{t^{2}+\mu^{2}}}-1\right]}
$$

If we change variables to $\mu s=\sqrt{t^{2}+\mu^{2}}$ we obtain

$$
S_{e}=-2 \int_{1}^{\infty} d s \frac{1}{\sqrt{s^{2}-1}} \frac{1}{e^{2 \pi \mu \alpha_{r} s}-1},
$$

which is a closed expression for the $O\left(e^{-\mu}\right)$ terms of the sum (C.2). The exponential corrections to the $\mu$-deformed Gamma-functions are readily obtained from (C.1). Incidentally an equivalent formula to (D.13) appears in [1].

\section{D.1. Conjecture for subsequent exponential corrections}

In analogy to the derivation of the first term in the exponential corrections one should be able to compute the full series (4.14). There are various subtleties in determining this, in particular related to the approximation of the $O(1 / \mu)$ term in (D.7). We shall now present some arguments which allow us to conjecture the exact expression for the leading 
order terms, i.e., of $O(1 / \mu)$. So, we wish to compute the expansion of the following term into a series of exponential corrections $O\left(e^{-2 \pi n\left|\alpha_{r}\right| \mu}\right)$

$$
\int_{\mu}^{\infty} d \mu \frac{\mu}{\omega_{z}} \sum_{n=1}^{\infty} K_{0}\left(2\left|\alpha_{r}\right| \mu n \pi\right)
$$

First, recall the asymptotic series for the Kelvin function ([35], VII., 7.34)

$$
K_{0}(x)=\sqrt{\frac{\pi}{2 x}} e^{-x}\left(\sum_{m=0}^{p-1} \frac{c_{m}}{(2 x)^{m}}+(-1)^{p} R_{p}\right)
$$

where the error term $R_{p}$ for large $p$, such that $x=p / 2+\sigma$ with $|\sigma|<1$, is given by

$$
R_{p} \sim 2 \sqrt{\frac{x}{\pi}} \frac{e^{-2 x}}{p}\left(\frac{1}{2}+O\left(\frac{1}{p}\right)\right) .
$$

Applying this to the Kelvin function appearing in (D.14), implies that

$$
\log \Gamma_{\mu}^{(r)}(z)_{\exp }=\sum_{n=1}^{\infty} e^{-2 \pi n\left|\alpha_{r}\right| \mu} \frac{\sqrt{\left|\alpha_{r}\right| \mu}}{2 \pi\left|\alpha_{r}\right| \sqrt{n} \omega_{z}}\left(1+O\left(\frac{1}{\mu}\right)\right)
$$

where it may be of use to point out that the $R_{p}$-terms do not contribute to the leading term in $1 / \mu$, and thus can be disregarded. If it now can be ensured that $O(1 / \mu)$ does not contain terms like $e^{-\mu}$ then we can retain the first term, as there is no mixing at the same order of the coefficient. However, to make this statement precise, a better approximation of the $O(1 / \mu)$ terms in $(\overline{\mathrm{D} .7})$ would have to be derived. 


\section{References}

[1] D. Berenstein, J. M. Maldacena, H. Nastase, Strings in flat space and pp waves from $N=4$ super Yang Mills, JHEP 0204, 013 (2002); hep-th/0202021.

[2] R. R. Metsaev, Type IIB Green-Schwarz superstring in plane wave Ramond-Ramond background, Nucl. Phys. B 625, 70 (2002); hep-th/0112044.

[3] R. R. Metsaev, A. A. Tseytlin, Exactly solvable model of superstring in plane wave Ramond-Ramond background, Phys. Rev. D 65, 126004 (2002); hep-th/0202109.

[4] M. B. Green, J. H. Schwarz, Superstring Field Theory, Nucl. Phys. B 243, 475 (1984).

[5] M. B. Green, J. H. Schwarz, Superstring Interactions, Nucl. Phys. B 218, 43 (1983).

[6] M. B. Green, J. H. Schwarz, L. Brink, Superfield Theory Of Type II Superstrings, Nucl. Phys. B 219, 437 (1983).

[7] M. Spradlin, A. Volovich, Superstring interactions in a pp-wave background, Phys. Rev. D 66, 086004 (2002); hep-th/0204146.

[8] M. Spradlin, A. Volovich, Superstring interactions in a pp-wave background. II, JHEP 0301, 036 (2003); hep-th/0206073.

[9] A. Pankiewicz, More comments on superstring interactions in the pp-wave background, JHEP 0209, 056 (2002); hep-th/0208209.

[10] A. Pankiewicz, B. Stefanski, Pp-wave light-cone superstring field theory, Nucl. Phys. B 657, 79 (2003); hep-th/0210246.

[11] Y. H. He, J. H. Schwarz, M. Spradlin, A. Volovich, Explicit formulas for Neumann coefficients in the plane-wave geometry, Phys. Rev. D 67, 086005 (2003); hepth/0211198, v2.

[12] J. Gomis, S. Moriyama, J. w. Park, Open + closed string field theory from gauge fields; hep-th/0305264.

[13] B. J. Stefanski, Open string plane-wave light-cone superstring field theory; hepth/0304114; B. Chandrasekhar, A. Kumar, D-branes in pp-wave light cone string field theory, JHEP 0306, 001 (2003); hep-th/0303223.

[14] J. Lucietti, S. Schafer-Nameki, A. Sinha, On the exact open-closed vertex in plane-wave light-cone string field theory, to appear in Phys. Rev. D; hep-th/0311231.

[15] I. R. Klebanov, M. Spradlin, A. Volovich, New effects in gauge theory from pp-wave superstrings, Phys. Lett. B 548, 111 (2002); hep-th/0206221.

[16] M. x. G. Huang, Three point functions of $N=4$ super Yang Mills from light cone string field theory in pp-wave, Phys. Lett. B 542, 255 (2002); hep-th/0205311.

[17] C. S. Chu, V. V. Khoze, Correspondence between the 3-point BMN correlators and the 3-string vertex on the pp-wave, JHEP 0304, 014 (2003); hep-th/0301036.

[18] A. Pankiewicz, Strings in plane wave backgrounds, Fortsch. Phys. 51, 1139 (2003); hep-th/0307027. 
[19] J. C. Plefka, Lectures on the plane-wave string / gauge theory duality, Fortsch. Phys. 52, 264 (2004); hep-th/0307101.

[20] M. Spradlin, A. Volovich, Light-cone string field theory in a plane wave; hepth/0310033.

[21] D. Sadri, M. M. Sheikh-Jabbari, The plane-wave / super Yang-Mills duality; hepth/0310119.

[22] R. Russo, A. Tanzini, The duality between IIB string theory on pp-wave and $N=4$ SYM: A status report; hep-th/0401155.

[23] M. B. Green, J. H. Schwarz, E. Witten, Superstring Theory. Vol. 2: Loop Amplitudes, Anomalies And Phenomenology, Cambridge University Press (1987).

[24] J. H. Schwarz, Comments on superstring interactions in a plane-wave background, JHEP 0209, 058 (2002); hep-th/0208179.

[25] O. Bergman, M. R. Gaberdiel, M. B. Green, D-brane interactions in type IIB planewave background, JHEP 0303, 002 (2003); hep-th/0205183.

[26] T. Takayanagi, Modular invariance of strings on pp-waves with $R R$-flux, JHEP $\mathbf{0 2 1 2 ,}$ 022 (2002); hep-th/0206010.

[27] M. R. Gaberdiel, M. B. Green, The D-instanton and other supersymmetric D-branes in IIB plane-wave string theory, Annals Phys. 307, 147 (2003); hep-th/0211122.

[28] M. R. Gaberdiel, M. B. Green, S. Schafer-Nameki, A. Sinha, Oblique and curved Dbranes in IIB plane-wave string theory, JHEP 0310, 052 (2003); hep-th/0306056.

[29] A. Sinha, N. V. Suryanarayana, Tadpole analysis of orientifolded plane-waves, JHEP 0211, 026 (2002); hep-th/0209247.

[30] V. Balasubramanian, M. x. Huang, T. S. Levi, A. Naqvi, Open strings from $N=4$ super Yang-Mills, JHEP 0208, 037 (2002); hep-th/0204196.

[31] D. Berenstein, E. Gava, J. M. Maldacena, K. S. Narain, H. Nastase, Open strings on plane waves and their Yang-Mills duals, hep-th/0203249.

[32] D. Berenstein, Shape and holography: Studies of dual operators to giant gravitons, Nucl. Phys. B 675, 179 (2003); hep-th/0306090.

[33] E. T. Whittaker, G. N. Watson, A Course of Modern Analysis, Cambridge University Press (1927).

[34] I. S. Gradshteyn, I. M. Ryzhik, Tables of Integrals, Series, and Products, Academic Press (2000).

[35] G. N. Watson, A Treatise on the theory of Bessel functions, Cambridge University Press (1966). 